\title{
DWORKIN Y EL UTILITARISMO: ALGUNAS INCONSISTENCIAS
}

\author{
I
}

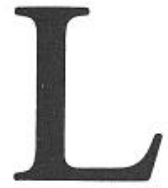

a estrategia de Dworkin al preparar sus trabajos parece seguir un modelo curiosamente similar. Comienza eligiendo una tesis como objetivo, luego la critica - exponiendo sus deficiencias - y concluye proponiendo su propia tesis alternativa. En sus primeros artículos, el objetivo fue la teoría de Hart sobi 2 el concepto de derecho '. Otras veces, el blanco fue la teoría de Posner sobre el análisis económico del derecho ${ }^{2}$. Pero, principalmente, su ataque se ha concentrado sobre el utilitarismo. Al respecto, creo que Dworkin ha presentado, en diferentes trabajos, distintas descripciones de la teoría utilitarista que no son mutuamente consistentes. Para poner ésto de relieve, aún a riesgo de resultar aburrido, debo citar con cierta extensión algunos de los pasajes en los que ha tratado el tema.

La crítica más importante que Dworkin formula al utilitarismo aparece en su libro Taking Rights Seriously ${ }^{3}$. Veamos los párrafos más relevantes.

«Si alguien tiene un derecho a algo, entonces es incorrecto para el gobierno el denegárselo, incluso aunque fuera en el interés general el hacerlo. Este sentido de un derecho (que podrá ser llamado el concepto anti-utilitarista de un derecho) me parece muy próximo al concepto de derecho empleado principalmente en escritos y argumentos políticos y jurídicos en años recientes». (Pag. 269) «Si la restricción sobre la libertad es lo suficientemente seria o severa, entonces es verdaderamente cierto que el gobierno no está autorizado a imponer esa restricción simplemente porque ella sería de interés general; el gobierno no está autorizado para restringir la libertad de palabra, por ejemplo, cada vez que creyera que ello mejoraría el bienestar general». (Pags. 269/270)

«Si no hay un derecho general a la libertad, entonces, ¿por qué los ciudadanos en una democracia tienen derecho a cualquier tipo específico de libertad, como la libertad de palabra, o de religión, o de actividad política?. No es respuesta el decir que si los individuos tienen estos derechos, entonces la comunidad como un todo estará mejor a largo plazo. Esta idea - que los derechos individuales pueden conducir a la utilidad de todos - puede o no ser verdadera, pero es irrelevante para la defensa de los derechos como tales, porque cuando decimos que alguien tiene derecho a hablar con franqueza, en el sentido político relevante, significamos que está auto-

' Cfr. "Is Law a System of Rules?», en Essays in Legal Philosophy, ed. por Robert S. Summers, Oxford, Basil Blackwell, 1970, pags. 25/60 y "Social Rules and Legal Theory", Yale Law Journal, Vol. 81, n. 5, pag. 855/889.

2 "Is Wealth a Value?", Journal of Legal Studies, Vol. 9, (1980), pags 191/226.

${ }^{3}$ London, Duckworth, 1.977. Cfr. capitulo 12, "What Rights Do We Have?». Las referencias siguientes corresponden a esta versión. Hay traducción castellana, Los derechos en serio, Barcelona, Ariel, 1.984. 
rizado a hacerlo incluso si ésto no resultara en el interés general». (Pag. 271)

«Los argumentos utilitaristas acerca de políticas... no suponen que cualquier forma de vida es inherentemente más valiosa que cualquier otra, sino que basan su pretensión - de que las restricciones a la libertad son necesarias para promover alguna meta colectiva de la comunidad - simplemente en el hecho de que ocurre que esa meta es deseada más amplia o profundamente que cualquier otra. Los argumentos utilitaristas de política, en consecuencia, parecen no oponerse - sino por el contrario abarcar - el derecho fundamental de igual consideración y respeto: porque tratan los deseo; de cada miembro de la comunidad a la par con los deseos de cualquier otro, con ningún aumento o disminución que refleje el punto de vista de que algún miembro es más o menos digno de consideración, o sus puntos de vista más o menos dignos de respeto, que cualquier otro. Esta apariencia de igualitarismo ha sido, creo, la principal fuente del gran atractivo que el utilitarismo ha tenido durante el último siglo, como filosofía política general». (Pag. 275) "Los argumentos utilitaristas se fijan en el hecho de que una restricción particular sobre la libertad hará feliz a más gente, o satisfará más de sus preferencias, según esté en juego el utilitarismo sicológico o de preferencias. Pero la preferencia total de la gente por una política mas que por otra puede ser vista como incluyendo, en una análisis adicional, tanto las preferencias que son personales, porque enuncian una preferencia para la asignación de un conjunto de bienes u oportunidades para el individuo mismo, cuanto las preferencias que son externas, porque enuncian una preferencia para una asignación de bienes u oportunidades para otros. Pero un argumento utilitarista que asigna peso crítico a las preferencias externas de los miembros de la comunidad no será igualitario en el sentido considerado. No respetará el derecho de todos a ser tratados con igual consideración y respeto». (Pag. 275) «Si los argumentos utilitaristas de política van a ser usados para justificar restricciones sobre la libertad, entonces debe tenerse cuidado en asegurar que los cálculos utilitaristas en los cuales el argumento está basado se fijen solo en las preferencias personales e ignoren las externas... Sin embargo, importante como es esa conclusión a nivel de la filosofía política, es en sí misma de limitada significación práctica, porque será imposible diseñar procedimientos políticos para discriminar con exactitud preferencias personales y externas... Más aún, las preferencias personales y externas están a veces tan inextrincablemente combinadas, que la discriminación es tanto sicológica cuanto institucionalmente imposible». (Pag. 276) «Deseo ahora proponer la siguiente teoría general de derechos. El concepto de un derecho político individual, en el sentido antiutilitarista fuerte que he distinguido antes, es la respuesta a los defectos filosóficos de un utilitarismo que computa las preferencias externas y a la imposibilidad práctica de un utilitarismo que no lo hace». (Pag. 277) 


\section{II}

La que Dworkin expone aquí es una versión correcta del utilitarismo; en ella aparecen a la luz las virtudes y los defectos de la teoría. Entre los defectos, el más notorio es que el utilitarismo está obligado a computar todas las preferencias, personales y externas, sin someterlas a ninguna valoración independiente. Porque el único criterio de valoración es el grado de felicidad o infelicidad que la satisfacción de las preferencias produzca. No hay criterio externo de valoración que nos permita decir que son mejores (o más valiosas) las preferencias (personales o externas) de la Madre Teresa de Calcuta que las preferencias de un jefe de la mafia. Ambas se computan por igual en el cálculo utilitarista, y esta circunstancia tal vez haya privado de varios adherentes al utilitarismo, y motivado teorías como la del propio Dworkin, basadas en derechos y no en metas. Porque la teoría utilitarista exige que demos una consideración imparcial a los ideales de nosotros y de los demás. Si el deseo de un nazi de no tener judíos a su alrededor, dice Hare, es lo suficientemente intenso como para contrapesar todos los sufrimientos causados a los judíos mediante los arreglos necesarios para que no se encuentren a su alrededor, entonces ese deseo debería ser satisfecho. Hare piensa que el problema se supera sólo porque los fanáticos de este tipo son muy difíciles de encontrar, pero éste es un argumento empírico y no ético ${ }^{4}$.

Podemos llamar a esta versión de Dworkin, entonces, la Versión 1; ella dice que para el utilitarismo los deseos de un individuo son tan importantes como los deseos de cualquier otro, debiéndose tomar en cuenta tanto las preferencias personales cuanto las preferencias externas de los individuos (entre otros motivos, porque es imposible separarlas).

A la Versión 1 es posible aplicarle la afirmación del propio Dworkin de que el utilitarismo, como una teoría general del valor o de la justicia, es falso, y su actual impopularidad, bien merecida s. Y como defensor de esta versión es que Dworkin pudo decir que Bentham no es el padre del liberalismo, sino que es - de hecho- una vergüenza para los liberales ${ }^{6}$.

La propia teoría que Dworkin propone en reemplazo de la Versión 1 no está exenta de defectos, y uno de ellos fue puntualizado por Ten. Se trata, otra vez, de la distinción entre preferencias personales y preferencias externas. Supongamos que se trata de elegir entre construir un teatro o una pileta de natación. Muchas personas que no nadan disfrutan más sentándose alrededor de la pileta que yendo al teatro; pero todavía están expresando sus preferencias personales, porque no es necesario preferir la pileta al teatro sólo porque uno desea nadar. Pero supongamos ahora que muchos no nadadores también tienen preferencias personales por el teatro, pero tienen también preferencias externas al-

${ }^{4}$ R.M. HARE, "Ethical Theory and Utilitarianism», en Contemporary British Philosophy, ed. por H.D. Lewis, London, George Allen and Unwin, 1.976, pag. 121.

" "Is Wealth a Value?", cit., pag. 216.

${ }^{6}$ Afirmación de Dworkin al ser reporteado por BRIAN MAGEE, en Men of Ideas, Oxford University Press, 1.982, pags. 227/228. 
truístas respecto del disfrute de sus hijos cuando nadan, preferencias externas que son más fuertes que sus preferencias personales por el teatro. Dworkin permitiría la construcción de la pileta en el primer ejemplo, pero no en el segundo. Esto parece poco equitativo para con los padres altruístas, tan poco equitativo como lo sería -en otros contextos- la consideración de las preferencias externas. El problema, concluye Ten, no consiste en excluir las preferencias externas sino en el contenido de las preferencias externas que se incluyen?

Y Hart ha señalado otra dificultad en la teoría de Dworkin. ¿Cuál es el elemento que puede corromper al argumento utilitarista y al voto democrático?, pregunta Hart. Para Dworkin la respuesta consistiría en señalar que hay una denegación de la libertad, entendida como la denegación de igual consideración y respeto. Hart piensa que esta respuesta es errónea. Y es aún menos creíble cuando la denegación de la libertad es el resultado de un procedimiento de decisión utilitarista, o de voto mayoritario, en el cual la preferencia minoritaria derrotada ha sido sopesada, igual que las otras, y derrotada por los números. Dworkin interpreta la derrota como un mensaje que expresa: «usted y quienes lo apoyan son inferiores, no merecen igual consideración y respeto». Hart la interpreta como un mensaje que dice: «usted y quienes lo apoyan son demasiados pocos. Usted ha sido contado como uno, pero no como más de uno. Aumente su número y sus puntos de vista pueden triunfar»" 8 .

Conviene precisar, para concluir este apartado, el alcance de la distinción de Dworkin entre preferencias personales y externas, que él mismo esbozó con corrección.

Un punto de vista liberal común en filosofía política, dice Arrow, sostiene que las preferencias de un individuo son -o deben ser- referidas sólo a los efectos de las acciones sociales sobre él. Pero, advierte, no hay una manera lógica de distinguir una clase particular de consecuencias que pertenezcan a un individuo dado. Así como no podemos descomponer las acciones sociales de modo de distinguir los componentes que pertenecen a un individuo dado, no podemos descomponer de un modo significativo las consecuencias de las acciones sociales en consecuencias separables para los miembros individuales de la sociedad.

De modo que desde un punto de vista fáctico, la distinción de Dworkin parece difícil de ser llevada a la práctica. La única defensa racional de la posición liberal, expresa Arrow, es que ella en sí misma es un juicio de valor. Los juicios de valor en favor de una preferencia social limitada deben ser computados como parte de los sistemas de valores que los individuos emplean al juzgar las acciones sociales alternativas 9 . De modo que se trata, entonces, de una distinción normativa. Lo que la sostiene es que, sea ella o no fácil de poner en práctica, es valioso que cuenten sólo las preferencias personales.

${ }^{7}$ C.I. TEN, Mill On liberty, Oxford, Clarendon Press, 1.980, pag. 32.

${ }^{8}$ H.L. HART, «Between Utility and Rights», en The Idea of Freedom, ed. por Alan Ryan, Oxford University Press, 1.979, pags. 93/94.

${ }^{9}$ KENNETH J. ARROW, "Values and Collective Decision-Making», en Philosophy Politics and Society, Third Series, ed. por Peter Laslett y W.G. Runciman, Oxford, Basil Blackwell, 1.978, pags. 222/223. 


\section{III}

Pero lo interesante es que, en un trabajo posterior, ${ }^{10}$ Dworkin presenta otra caracterización del utilitarismo que tiene dos puntos salientes:

1.) No recoge adecuadamente, a diferencia de la Versión 1, los rasgos relevantes de la teoría utilitarista.

2.) Es inconsistente con la propia Versión 1. De nuevo voy a citar los párrafos más característicos en los que esta versión es expuesta.

«Imaginemos una comunidad de muchas personas, incluyendo Sara. Si la Constitución establece una versión del utilitarismo que estipula que las preferencias de Sara deben contar el doble que las de los otros, esta sería una versión inaceptable, no igualitaria, del utilitarismo. Pero supongamos ahora que la claúsula constitucional es la forma común del utilitarismo, esto es, que ella es neutral respecto de todas las personas y sus preferencias, pero que un número sorprendente de personas quiere mucho a Sara, y -en consecuencia- prefiere fuertemente que sus preferencias cuenten el doble que las de los otros en las decisiones políticas diarias efectuadas según los cálculos utilitaristas. Cuando Sara no recibe lo que ella debería tener si sus preferencias contaran el doble que las de los demás, entonces esa gente será infeliz, porque sus preferencias especiales surgidas de su amor por Sara serán inclumplidas. Si se computan estas preferencias especiales, en consecuencia, Sará recibirá mucho más la distribución de bienes y oportunidades de lo que habría recibido de otra manera. Yo sostengo que esto derrota el matiz igualitario de la constitución utilitarista aparentemente neutral... En realidad, la provisión aparentemente neutral es entonces auto-socavada porque otorga un peso crítico - para decidir cual distribución promueve mejor la utilidad - a los puntos de vista de aquellos que sostienen la teoría profundamente no-neutral (algunos dirían utilitarista) de que las preferencias de alguien deberían contar más que las de los otros». (Pag. 202)

«Supongamos que en la comunidad hay un nazi, por ejemplo, cuyo conjunto de preferencias incluye la de que todos los arios tengan más preferencias cumplidas que los judíos, solo por ser quienes son. Un utilitarista neutral no puede decir que no hay ninguna razón en la moral política para rechazar o descalificar esa preferencia, para no descartarla como simplemente incorrecta, para no esforzarse por cumplirla con toda la dedicación que los órganos dedican al cumplimiento de cualquier otro tipo de preferencia. Porque el propio utilitarismo proporciona una razón: su tesis más funda-

${ }^{10}$ "Is There Right to Pornography?», Oxford.Journal of Legal Studies, Volume 1, 1.981. De aquí tomé las citas que siguen. 
mental es que las preferencias de las personas deberian ser sopesadas sobre una base igual en las mismas escalas, que la teoría nazi de la justicia es profundamente incorrecta y que los órganos deberían oponerse a la teoría nazi y esforzarse en derrotarla, y no en cumplirla. Un utilitarista neutral tiene de hecho prohibido, por razones de consistencia, el adoptar la misma actitud políticamente neutral respecto de la preferencia política del nazi que respecto de otro tipo de preferencias». (Pag. 203)

«Podríamos escapar a este punto, desde luego, distinguiendo dos formas diferentes - o niveles - de utilitarismo. La primera sería presentada simplemente como una teoría débil acerca de como debería ser elegida una constitución política en una comunidad cuyos miembros prefieren diferentes tipos de teorías políticas. La segunda sería una candidata para la constitución que va a ser así elegida; podría argumentar en favor de una distribución que maximizara la satisfacción agregada de las preferencias personales en la distribución real de los bienes y oportunidades, por ejemplo. En ese caso, la primera teoría argüiría sólo que a las preferencias del nazi debería serle concedido igual peso que a las preferencias del segundo tipo de utilitarismo en la elección de una constitución, porque cada una de ellas está igualmente autorizada a obtener la constitución que prefiere, y no habría ninguna contradicción en esa proposición. Pero, por supuesto, la teoría utilitarista neutral que estamos ahora considerando no es simplemente una teoría débil de ese tipo. Ella propone una teoría de la justicia como una constitución política completa, no simplemente una teoría acerca de como elegir una constitución, y así no puede escapar a la contradicción de la modestia». (Pag. 204)

Llamemos a esta la Versión 2; ella dice que el utilitarista debe distinguir entre las preferencias que deben ser tomadas en cuenta (aparentemente, en esta versión no hay dificultad en separar las preferencias personales de las que no lo son).

\section{IV}

De esta última descripción ya no puede decirse que sea una versión correcta del utilitarismo. Si la sociedad es más feliz en caso de que Sara reciba un doble voto en la consideración de todos los asuntos de la comunidad, el utilitarismo recomendaría (u ordenaría) darle a Sara un doble voto. Si un órgano del estado rehusara tomar en cuenta las preferencias del nazi en el cálculo de la felicidad, no obraría como un órgano utilitarista. Una cosa es decir que la teoría nazi de la justicia es profundamente incorrecta (porque no ocasiona la mayor felicidad del mayor número) y otra muy distinta es descartar por tal causa las preferencias del nazi para el cálculo de la mayor felicidad del mayor número. Un utilitarista consistente tiene que computar todas las preferencias. No puede seleccionar las preferencias sobre la base de su mérito, porque su único 
criterio del mérito es la consecución de la mayor felicidad del mayor número.

El utilitarismo, en la Versión 2, tornaría innecesaria la teoría de derechos del propio Dworkin (que él, en la Versión 1, calificaba de antiutilitarista). En la Versión 1, el utilitarismo, aparentemente, abarcaba el derecho a igual consideración y respeto. Pero sólo aparentemente; en la realidad no lo hacía, precisamente por tomar en cuenta tanto las preferencias externas cuanto las personales.

Tampoco puede decirse que la Versión 2 sea compatible con la Versión 1. En la Versión I el nazi contaba; en la Versión 2, no debe contar. En la Versión 1, no hay manera de separar las preferencias personales de las externas; en la Versión 2 deben separarse (y ésto es importante en la práctica, a pesar del carácter normativo de la distinción). Varios de los aspectos que recomendaban el rechazo del utilitarismo en su Versión $l$, no son ya aspectos del utilitarismo en la Versión 2. Yo creo que la Versión I es la correcta; los puntos débiles del utilitarismo que aparecen en ella son genuinos puntos débiles. La Versión 2 presenta al utilitarismo bajo un ángulo mucho más atractivo, pero ya deja de ser utilitarismo.

Quiero señalar brevemente otros defectos de la Versión 2, aparte de su incompatibilidad con la Versión $l$ y de sus características distorsionantes respecto de la teoría utilitarista.

Según Dworkin, para que el utilitarismo fuera neutral tendría que tomar en cuenta sólo las preferencias neutrales (dejemos de lado, en este caso, el problema de si es posible separar unas preferencias de las otras). Me parece que Dworkin incurre aquí en una confusión de niveles, que puede representarse sencillamente mediante este diagrama:
NIVEL 1
NIVEL 2
Principio Utilitarista Neutral
Preferencias

La neutralidad aparece sin duda en el NIVEL 1, puesto que se le exige al utilitarista que sea neutral respecto de todas las preferencias que debe evaluar para efectuar el cálculo de la felicidad. Pero Dworkin quiere que la neutralidad vuelva a aparecer en el NIVEL 2, y esto semeja ser contradictorio. Si se le exige al utilitarista en el NIVEL 1 que sea neutral respecto de las preferencias del NIVEL 2, ésto implica que no debe efectuar selección alguna entre ellas, sino, por el contrario, evaluarlas por igual. Dworkin le pide, en cambio, que atienda solo a las preferencias neutrales. ¡Pero seleccionar a las preferencias neutrales no es ser neutral respecto de las preferencias! Todo lo contrario: es mostrar un fuerte prejuicio a favor de las preferencias neutrales.

Me parece que Dworkin intenta solucionar aquí, en forma rápida y no del todo convincente, el complejo problema de la tolerancia para con los intolerantes. Y lo hace de una manera más próxima a Popper que a Rawls. En ciertas consecuencias, Popper reclama - en nombre de la tolerancia - el derecho a no tolerar a los intolerantes. Exige que todo movimiento que predique la intolerancia quede al margen de la ley, y que se considere criminal cualquier incitación a la intolerancia y a la persecución ". Rawls, en cambio, es algo más benévolo: la libertad del in-

"KARL R. POPPER, La sociedad abierta y' sus enemigos, Buenos Aires, Paidos 1.981, pag. 512 . 
puede haber dos motivaciones básicas diferentes para optar por un punto de vista utilitarista: a) la idea de que el placer es bueno, lo que conduciría a la adopción del utilitarismo clásico. b) la creencia en un principio igualitario abstracto, que conduciría a adoptar una posición utilitarista compatible con la afirmación del postulado de igual consideración y respeto. Para Dworkin, el primer punto de vista es erróneo, puesto que conduce a comprometernos con una aumento indefinido de la población, sin tener en cuenta el nivel de vida promedio, crítica formulada en su momento por Rawls is.

Ante todo, la crítica de Rawls no puede ser considerada cómo una refutación absoluta del primer punto de vista, ni él pretende que lo sea. Se puede defender la idea de que el placer es bueno y rechazar, no obstante, la versión clásica, aceptando - en cambio - la idea de utilidad promedio, basada en el mismo fundamento que la anterior.

Pero veamos el fondo de la propuesta de Dworkin. Desde luego que, con esta distinción que formula, Dworkin evita las inconsistencias que he señalado. La Versión I sería una descripción del primer punto de vista y la Versión 2 una descripción del segundo punto de vista. Pero me parece que hay algo más que decir acerca de ésto. Lo más importante es que no entiendo como el segundo punto de vista puede ser considerado como una descripción del utilitarismo. Aquí pueden presentarse tres tipos de alternativas: 19) Un individuo defiende el principio igualitario abstracto porque piensa que es el principio que mejor conduce a la mayor felicidad (o placer) general. Este individuo es un utilitarista, por supuesto, pero un utilitarista que acepta el primer punto de vista, no el segundo. Si se le demuestra que el igualitarismo no conduce a la mayor felicidad del mayor número, él abandonará el principio igualitario. 2.) Un individuo defiende el principio igualitario abstracto porque piensa que produce las mejores consecuencias posibles, consecuencias que él no identifica - necesariamente - con la felicidad o con el placer. Este individuo es un consecuencialista, sin duda, pero no un utilitarista, en cualquier definición aceptable del término ${ }^{16}$. Porque es capaz de resignar la pérdida de felicidad para ganar un incremento en algún otro bien distinto de ella, y es contradictorio asignar esta posición a un utilitarista. 3.) Un individuo defiende el principio igualitario abstracto porque piensa que es un principio bueno en si mismo. Este es un deontologista, pero no puede ser nunca considerado un consecuencialista en ninguna de sus variantes (una de las cuales, precisamente, es el utilitarismo).

Sigo pensando, pues, que - a pesar de esta distinción sutil- las dos versiones de Dworkin son inconsistentes, y una de ellas, inadecuada para dar cuenta del utilitarismo.

is A Theory of Justice, cit., pags. 162/163.

${ }^{16}$ Cfr. DEREK PARFIT, Reasons and Persons, Oxford, Clarendon Press, 1.984, pags. $26 / 27$. 